\title{
LAS TECNOLOGÍAS DE INFORMACIÓN Y COMUNICACIÓN (TIC) DESDE UNA PERSPECTIVA SOCIAL
}

\author{
Esmeralda Sánchez Duartel \\ Investigadora del Instituto de Estudios Latinoamericanos de la Universidad Nacional \\ Heredia, Costa Rica
}

Recibido: 11 de setiembre 2007 • Aprobado: 20 de noviembre 2007

\begin{abstract}
Resumen: Este artículo analiza las potencialidades de las tecnologías de la información y comunicación a la luz de sus aportes en un contexto social y básicamente rural. La autora enfoca las ventajas de la apropiación social de este recurso, sus amenazas y riesgos, así como los ejes que deben valorarse cuando se pretende un impacto en el desarrollo social. Esta perspectiva permite el abordaje de la relación entre las TIC y preocupaciones sociales tan importantes como la desigualdad social, la equidad de género y la participación comunitaria, entre otras. El artículo finaliza planteando una reflexión sobre algunos desafíos en esta materia.
\end{abstract}

Palabras clave: TICs, tecnología, desarrollo, desarrollo social.

\begin{abstract}
The intention of the article is to announce the needs of a curriculum Identified in the NicaraguanThis article analyses the potential of information and communication technologies from a rural social context approach. The author approaches the advantages of the social incorporation of this resource, its menaces and risks as well as the aspects that have to be valued when an impact on social development is expected. This perspective allows tackling the relation between ICTs and social concerns such as social inequality, gender equity, and community participation, among others. The article ends up presenting a reflection about some challenges in this matter.
\end{abstract}

Key words: ICTs, technology, development, social development.

\section{INTRODUCCIÓN}

Con el fin de hacer más breve su referencia, las tecnologías de la información y la comunicación suelen ser denominadas TIC.

Se han elaborado múltiples definiciones en torno a lo que son las TIC, muchas de las cuales resultan ser muy generales, en tanto otras incluyen aspectos más precisos. Un acercamiento bastante

Máster en Educación Rural Centroamericana. Académica del Instituto de Estudios Latinoamericanos de la Universidad Nacional. Ha laborado en la Universidad Estatal a Distancia. Sus principales intereses profesionales giran en torno a la educación y los asuntos indígenas.

Correo electrónico: e_sanchezd@hotmail.com,esme.sd@gmail.com 
amplio es el del portal de la Sociedad de la Información de Telefónica de España, citado por (Daccach, J. C. (s.f., p. 1) que indica:

Las TIC (Tecnologías de la Información y Comunicaciones) son las tecnologías que se necesitan para la gestión y transformación de la información, y muy en particular el uso de ordenadores y programas que permiten crear, modificar, almacenar, proteger y recuperar esa información.

En este caso, los ordenadores o computadoras son fundamentales para la identificación, selección y registro de la información. De modo particular, subyace un sentido social en el uso de la tecnología, al asociarla a la comunicación, quehacer humano en el cual ineludiblemente se insertan las relaciones sociales.

Una definición más específica es la del Programa de las Naciones Unidas para el Desarrollo (PNUD, 2002), en el Informe sobre el Desarrollo Humano en Venezuela, citado por Daccach (s.f., p. 1):

...Las TIC se conciben como el universo de dos conjuntos, representados por las tradicionales Tecnologías de la Comunicación (TC) -constituidas principalmente por la radio, la televisión y la telefonía convencional- y por las Tecnologías de la Información

(TI) caracterizadas por la digitalización de las tecnologías de registros de contenidos (informática, de las comunicaciones, telemática y de las interfaces).

Dicha concepción es significativa, porque no sólo incluye a las modernas tecnologías, sino también a los medios de comunicación social convencionales; la radio, la televisión y el sistema telefónico. Desde esta perspectiva, más amplia e inclusiva, es más factible considerar los contextos rurales, ya que en muchos de ellos aún imperan esos medios tradicionales de comunicación, y solo paulatinamente se han podido incorporar las TIC más recientes, sobre todo la Internet.

En esta época se vuelve importante porque, en términos generales, la sociedad moderna se caracteriza por el veloz despliegue y desarrollo de la tecnología y la ciencia, así como por la globalización de la información.

\section{LAS TIC DESDE LA PERSPECTIVA SOCIAL}

De manera definitiva, la introducción de las tecnologías de la información y la comunicación ha traído consigo cambios significativos en la sociedad. La puesta en práctica de las TIC afecta a numerosos ámbitos de la vida humana, en términos teóricos y de gestión cotidiana. La pregunta que surge entonces es: ¿deben estar las tecnologías de la comunicación al servicio del desarrollo humano, o debe éste sujetarse a los "vaivenes" y "caprichos" de la tecnología?

Aunque no hay una respuesta única ni contundente para la interrogante anterior, sí consideramos factible hacer algunas reflexiones en este sentido. Como observación preliminar, diremos que el desarrollo de una comunidad (local o nacional) no se limita a la posibilidad de tener o no conectividad; lo relevante es que dicha conectividad sea con sentido, equitativa y que la apropiación de los recursos sea social; ello permite sacar un mayor provecho de las oportunidades e incrementar los potenciales resultados positivos. 
El uso con sentido apunta a la posibilidad de utilizar efectivamente las TIC, así como saber combinarlas con otras formas de comunicación social. Incluye también la eventualidad de producir contenidos propios, o bien, de acceder a contenidos de otros que resulten útiles. Es armonizar adecuadamente el recurso Internet con otros, como la radio comunitaria, las reuniones presenciales, los materiales impresos y los videos.

Las TIC deben aprovecharse para el desarrollo integral de una comunidad. Una visión integral de desarrollo no implica que se apunte solo hacia el crecimiento económico sino, sobre todo, que impulse el potencial humano en sus diferentes dimensiones para afianzar así la prosperidad económica pero con equidad, y el fortalecimiento democrático con transparencia y justicia social.

Es preciso considerar que las TIC no son neutras, positivas o negativas; son simplemente lo que el usuario haga de ellas; no obstante, si quedan oscilando en la nada, pueden favorecer las desigualdades sociales, por lo que es preferible asumirlas con responsabilidad y darles una orientación positiva en beneficio del desarrollo integral de las comunidades.

Las tecnologías de la información y la comunicación no son suficientes ni imprescindibles para que se dé el desarrollo humano; lo cierto es que, difícilmente vinieron para no marcharse, por lo que se torna urgente encausarlas para que asuman un papel social al servicio del desarrollo de los pueblos y, ante todo, de los sectores más necesitados.

Se entiende que las TIC no siempre son relevantes para transformar la realidad. El desafío consiste más bien en discernir cuándo y en qué condiciones pueden aportar al desarrollo. El acceso a las TIC no soluciona con su sola presencia el problema del desarrollo humano, sino que es necesario ir más allá de la conectividad, promoviendo el acceso equitativo, uso y apropiación social de los recursos disponibles.

Por otro lado, la brecha digital amenaza hoy con incrementar las brechas sociales; esto significa que tenemos que repensar el potencial de las TIC como herramientas que pueden ayudar a construir sociedades más justas, equitativas y democráticas.

Hasta aquí tenemos que algunos de los elementos centrales de la visión social de las TIC serían:

- Ir más allá de la conectividad.

- Propiciar condiciones favorables en el entorno social.

- Minimizar las amenazas y riesgos.

- Potenciar resultados positivos.

\section{El acceso equitativo}

Por acceso equitativo estamos entendiendo la disponibilidad de conectividad a costos razonables y al alcance de los sectores económicamente menos favorecidos, así como de la capacitación básica en el manejo de las herramientas, para que más personas puedan utilizarlas independientemente del género, origen social, etnia o credo. Esto evita que las TIC se conviertan en un factor de exclusión para los sectores sociales menos favorecidos.

\section{Apropiación social de las TIC}

Los recursos de Internet deben ayudar a resolver problemas concretos. La apropiación consiste en los cambios que las TIC producen para el bien social. Cuando se tornan en herramientas 
útiles para transformar la realidad en beneficio del ser humano, las TIC contribuyen al desarrollo social, esto sucede, por ejemplo, cuando se mejora la calidad de la educación con el uso de recursos pedagógicos innovadores.

La introducción de las TIC no garantiza el desarrollo, pues ellas se vienen a insertar en contextos que ya poseen en sí mismos desigualdades de todo tipo, que las TIC no modifican automáticamente. Solo condiciones propicias previas que apunten a la igualdad social, relativas al contexto, pueden garantizar que las TIC lleguen a favorecer el desarrollo social.

Es necesario que las TIC se inserten en prácticas sociales ya existentes de personas, grupos u organizaciones; de este modo, servirán como herramientas que potenciarán el trabajo en un mundo real y concreto, y no a la inversa, no se trata de promover y forzar la realización de acciones con el fin de utilizar las TIC.

También debe considerarse que las TIC sean parte de una estrategia de comunicación coherente, de lo contrario, las organizaciones se estancan resolviendo problemas técnicos, de conexión y de manejo de tecnologías siempre cambiantes. En este caso, en lugar de ayudar a resolver problemas, se convierten en un obstáculo.

El uso de las TIC no necesariamente promueve la participación ciudadana; la democracia debe estar presente desde antes de las TIC, o bien, desde el inicio de su utilización. La participación debe darse desde la identificación de los problemas, y en la construcción de las formas para solucionarlos; esto ayuda al empoderamiento y la sostenibilidad futura de los proyectos.

El uso de las TIC también debe inscribirse en la ética de la solidaridad, la reciprocidad y el entusiasmo, lo cual debe fortalecerse de manera creativa y firme.

\section{Amenazas y riesgos en el uso de las TIC}

De igual modo, es importante estar alerta ante las posibles consecuencias negativas que el uso de las TIC pueden traer para el desarrollo y, frente a estas posibles amenazas y riesgos, diseñar estrategias que las disminuyan o minimicen. Algunas de estas amenazas o riesgos por considerar son:

\section{Aumento de las desigualdades}

Internet es un medio potencial para aumentar las desigualdades sociales, económicas, culturales y de distinta índole, así como para hacer que las nuevas oportunidades se distribuyan solo entre quienes tienen acceso a ésta.

\section{Homogeneización o imposición}

Contenidos, idioma y cultura, entre otros factores que se privilegian en la Internet, pueden ser negativos al inclinarse, voluntaria o involuntariamente, a uniformar ideas, preferencias y visiones de mundo; descartando o dejando de lado las particularidades de otros pueblos. 


\section{Abundancia descontrolada e inmovilización}

Más información no necesariamente equivale a mayor conocimiento. Se corre el riesgo de banalizar o dar una importancia superflua a la información a la que se tiene acceso; al "consumirla" sin analizarla ni reflexionarla. Por otra parte, en lugar de mejorar la vida de la gente, las TIC pueden causar sobrecarga de trabajo, estrés, consumismo y, en general, un lamentable deterioro en la calidad de vida.

\section{Aislamiento y fragmentación}

Las TIC pueden provocar separación y aislamiento. Hay personas y grupos que reducen cada vez más sus intereses y su quehacer cotidiano a pequeños claustros de intercambio cibernético. Solo una ciudadanía informada, organizada y capaz de apropiarse responsable y equitativamente de los recursos de la Internet, puede hacer frente a las amenazas que implica la introducción de las TIC en la sociedad.

Junto a estos riesgos, es indudable que el uso de las TIC trae o puede traer resultados positivos, como el simplificar y agilizar el acceso a más fuentes de información actualizada; el incremento de formas de intercambio rápidas y a un bajo costo, que permitan la apertura de nuevas ventanas más allá de lo local, el fortalecimiento de una participación consentida e informada, tanto de organizaciones y grupos de la sociedad civil, como de investigadores e investigadoras; quienes rompen así barreras geográficas para ser parte de diálogos, aprendizajes e intercambios, abren la posibilidad de establecer nuevas formas de trabajo colaborativo, redes y alianzas.

Así, las TIC pueden ayudar a personas y organizaciones a fortalecer su imagen y su autoestima; a abrirse espacio y dar a conocer al mundo sus intereses y prioridades. En el campo de la educación, las TIC tienen la posibilidad de aportar a la formación; pueden incentivar a muchas personas para que regresen a estudiar y para que mejoren la lectura y la escritura, entre otras alternativas. En términos generales, las TIC pueden devenir en un apoyo para que muchos se sientan parte activa y capaz de una sociedad que sistemáticamente les ha excluido y explotado, es el caso, por ejemplo, de los pueblos indígenas.

Puntualicemos ahora en algunos aspectos que podrán ser tomados en cuenta para aprovechar las TIC desde la perspectiva del desarrollo social:

\section{Valores}

- Cultivar la diversidad y fomentar la inclusión respetando las diferencias culturales, étnicas, de género, otros.

- Tomar en cuenta las diferentes voces de la sociedad civil o de la comunidad.

- Formular políticas apropiadas y contextualizadas.

\section{Estilos de trabajo}

- Fomentar el trabajo colaborativo para sacar provecho de los recursos disponibles. 
- $\quad$ Fortalecer las capacidades locales formando usuarios críticos y no solo consumidores pasivos de información.

- Reflexionar colectivamente.

\section{Estrategias}

- Trabajar en diferentes niveles; en el local, nacional e internacional.

- Mantener redes efectivas para hacer dinámico el intercambio de información y de experiencias.

- $\quad$ Fortalecer las alianzas con el sector público y privado, así como con las organizaciones de la sociedad civil.

- $\quad$ Incidir en la formulación de políticas que propicien el uso y apropiación de estas herramientas.

\section{Ejes para la colaboración}

- $\quad$ Promover una visión social de la Internet, que trascienda la conectividad.

- Promover entornos con condiciones que favorezcan el acceso y uso democrático y equitativo de las TIC, para todos y todas.

- Realizar acciones concretas que pongan en práctica la colaboración.

- Mejorar el acceso comunitario a Internet.

- Aprovechar la convergencia de medios de comunicación para obtener mayor impacto.

- Aprender de resultados e impactos.

\section{En el ámbito de la comunidad}

- Considerar si los centros de cómputo que hay en la comunidad responden a las necesidades más sentidas de ésta.

- $\quad$ Fortalecer la capacidad de estos sectores para que saquen provecho de los recursos de Internet. La Internet puede contribuir a fortalecer su identidad y a tener una mayor participación en el mundo.

- Construir de forma colectiva y permanente, nuevas visiones, capacidades, conocimientos y procedimientos de trabajo.

- Participar en la formulación de políticas integrales e incluyentes, que contribuyan a convertir a las TIC en herramientas efectivas para el desarrollo humano.

Perspectiva de género

- $\quad$ Acciones que promuevan la igualdad de oportunidades en el uso, acceso y apropiación de las TIC.

- Mecanismos que compensen las desigualdades existentes en la sociedad y que contribuyan a transformarlas. 
- Considerar los intereses estratégicos de las mujeres.

- Generación permanente de datos relativos al acceso, uso y apropiación de las TIC, desagregados por género.

- Utilización de las TIC para mejorar la calidad de vida de las mujeres dentro y fuera del ámbito doméstico.

- Fortalecimiento de la perspectiva de género en las políticas públicas locales, nacionales e internacionales, relacionadas con las TIC.

\section{Algunas ideas para un plan de acción que optimice el uso de las TIC en espacios educativos}

- $\quad$ Readecuación de políticas y planes del sistema educativo a nivel nacional, para evitar la pérdida de la identidad de los pueblos con el uso y abuso de las TIC.

- Socializar las experiencias de coordinación interinstitucional existentes, destacando sus resultados positivos, para propiciar el aprendizaje colectivo.

- Promover reestructuración de los sistemas educativos para ir incorporando las tecnologías de la comunicación y la información, como herramientas para favorecer el desarrollo social integral y equitativo en todos los sectores de la sociedad.

- Generar diagnósticos técnicos de necesidades de capacitación y formación profesional.

- Formar maestros y maestras que reproduzcan y apoyen el uso con sentido y responsabilidad de las TIC y trabajar a corto plazo en procesos de sensibilización.

- Expandir a nivel nacional, e incluso de la región centroamericana, las experiencias locales.

- Construir una propuesta de educación con participación de los sectores más vulnerables y excluidos social y económicamente.

- Elaborar una propuesta educativa que articule los conocimientos de los distintos pueblos que conforman el mosaico de nuestras nacionalidades centroamericanas, en la que impere el respeto a las particularidades de cada pueblo.

- $\quad$ Promover más que un beneficio individual, un beneficio colectivo.

- Construir un sistema en donde la misma enseñanza brinde herramientas teóricas y metodológicas que contribuyan al acompañamiento y monitoreo de las realidades y necesidades comunitarias.

- Generar un programa de capacitación con temáticas de carácter general en una primera etapa y posteriormente brindar la posibilidad de especialización en temáticas específicas, que responda a la demanda de la población.

- Diversificar las propuestas, no solo incluir las de una entidad, con el objetivo de maximizar las experiencias, los recursos, o sea, propiciar acciones integradoras donde todos los resultados alcanzados se aprovechan y comparten.

- Aprovechar aliados tales como las universidades, ministerios de educación, agencias de cooperación internacional, centros de formación profesional, organizaciones no gubernamentales, empresas privadas, entre otros.

\section{CONSIDERACIONES FINALES}

A manera de síntesis, cabe mencionar que el enorme potencial de las TIC debe aprovecharse en el marco de los principios y valores de la sociedad justa y equitativa que queremos construir. La 
forma en que se utilicen estos recursos debe garantizar pasos seguros hacia este ideal de sociedad. Para ello, es importante considerar la evaluación constante, pues como en cualquier otro campo, las TIC deben ser objeto de valoración, reflexión, realimentación y mejoramiento, todo lo cual se deriva de la evaluación periódica.

En esta perspectiva, entre los desafíos más importantes que nos plantea este tema está la vigilancia constante de la integración, de una visión social humanizada. Igualmente, debemos cuidar que este recurso se aproveche para generar conocimientos y capacidades nuevas, así como fortalecer alianzas entre la sociedad civil, la empresa privada y el gobierno, todo ello con miras a lograr que éstas verdaderamente impacten en el desarrollo social de las comunidades.

\section{REFERENCIAS}

Daccach, J. C. (s. f.). Tecnologías de la Información y Comunicaciones (TIC). Consultado el 12 de junio de 2007 de http://www.gestiopolis.com/delta/term/TER434.html

Wikipedia La Enciclopedia Libre. (s.f.). Tecnologías de información y comunicación. Concepto. Consultado el 12 de junio de 2007 de http://es.wikipedia.org/wiki/Tecnolog\%C3\%ADas_de_ la_informaci\%C3\%B3n 